\title{
BMJ Open Inclusion of diabetic retinopathy screening strategies in national-level diabetes care planning in low-income and middle-income settings: protocol for a scoping review
}

Katie Curran (D) , ${ }^{1}$ Prabhath Piyasena (D) , ${ }^{1,2}$ Nathan Congdon, ${ }^{1,3}$ Lisa Duke, ${ }^{4}$ Belma Malanda, ${ }^{4}$ Tunde Peto (1) ${ }^{1,5}$

To cite: Curran K, Piyasena P, Congdon N, et al. Inclusion of diabetic retinopathy screening strategies in national-level diabetes care planning in low-income and middleincome settings: protocol for a scoping review. BMJ Open 2020;10:e038647. doi:10.1136/ bmjopen-2020-038647

- Prepublication history and additional materials for this paper is available online. To view these files, please visit the journal online (http://dx.doi org/10.1136/bmjopen-2020038647).

Received 18 March 2020 Revised 07 August 2020 Accepted 10 August 2020
Check for updates

(C) Author(s) (or their employer(s)) 2020. Re-use permitted under CC BY-NC. No commercial re-use. See rights and permissions. Published by BMJ.

For numbered affiliations see end of article.

Correspondence to

Katie Curran;

kcurran12@qub.ac.uk

\section{ABSTRACT}

Introduction The diabetes mellitus (DM) epidemic is a major public health concern globally, with the highestburden in low-income and middle-income countries (LMICs). Diabetic retinopathy (DR) is a microvascular complication of diabetes, and if left untreated can lead to visual impairment and blindness. Epidemiological studies suggest that the incidence of sight-threatening DR is decreasing in high-income countries due to improved treatments and management of DM; however, these trends are not replicated in LMICs. In this paper, we outline a scoping review protocol that aims to identify which LMICs have included DR in their national DM, non-communicable disease or prevention of blindness plans. The scoping review also aims to assess gaps when implementing national DR screening programmes in LMICs.

Methods and analysis This scoping review will follow the Arksey and 0'Malley (2005) methodology and the Preferred Reporting Items for Systematic Review and Meta-Analysis extension for Scoping Review guidelines. A comprehensive search of peer-reviewed and grey literature will be conducted from October 1989 (St. Vincent Declaration) to February 2020. Studies will be identified from electronic databases; Medline, Embase and CENTRAL (Cochrane Library). To identify further relevant articles, a hand search will be conducted using the reference lists of included studies. Two reviewers will independently screen records for relevant data and disagreements about eligibility will be resolved through consensus or arbitration by a third reviewer. A quantitative analysis will be performed to highlight key findings and thematic analysis will be used to identify emerging themes and subthemes from included studies. The key themes will highlight countries progress in terms of national-level DR service planning and screening implementation.

Ethics and dissemination No ethical approval is required because the scoping review methodology aims to synthesise information from publicly available resources. The results will be disseminated through conference presentations and peer-reviewed publication.

\section{INTRODUCTION}

An estimated 463 million adults (20-79 years) are living with diabetes mellitus
Strengths and limitations of this study

- The organisation and development of diabetic retinopathy (DR) services in national-level diabetes mellitus care planning in low-income and middleincome countries (LMICs) will be explored, a topic which has received little attention.

- Countries that lack national-level DR planning will be identified, which is of use to funders and programme planners.

- There are a limited number of published studies meeting the inclusion criteria due to lack of publication of policy-related documents in LMICs.

- Non-English literature will not be included in this scoping review.

(DM) globally. ${ }^{2}$ By 2045, it is estimated that 700 million people will be living with DM, an increase of approximately $51 \%$ from 2019. ${ }^{2}$ The global burden of this condition poses a major public health challenge; therefore, urgent action is necessary at global, national and regional levels. Diabetic retinopathy (DR), including diabetic macular oedema, is one of the most common microvascular complications of DM and if left untreated can cause visual impairment and lead to blindness. ${ }^{3} 4$ Approximately $80 \%$ of people with DM reside in low-income and middle-income countries (LMICs), and the burden of sight-threatening DR in these countries is high. ${ }^{156}$ The expenditure of DR also places a significant burden on healthcare systems and the economy, especially in low-resource settings. ${ }^{7}$ Existing literature has demonstrated that proper management of DM and early detection and treatment through DR screening will reduce the incidence and progression of sight-threatening DR. ${ }^{8-10}$ 
Since the introduction of systematic DR screening in the UK, DR is no longer the leading cause of blindness among working-age adults with DM. ${ }^{11} 12$ As outlined by Piyasena et $a l,{ }^{13}$ lack of infrastructure and human resources are the main barriers to DR screening in LMICs. Implementing diabetic eye screening programmes (DESPs) involves high capital expenditure; therefore, understanding and overcoming any potential barriers may be crucial prior to the implementation of a programme. ${ }^{13}$ New strategies to address the rapid emergence of DR are needed and have been highlighted in the World Health Organisation (WHO) 'World Vision Report'. ${ }^{14}$ The report identified the specific need for health promotion, prevention and treatment strategies to be aligned to mitigate the impact of DR. ${ }^{14}$ Regular eye examinations, periodic screening and timely treatment are part of these strategies. ${ }^{14}$ The International Agency for the Prevention of Blindness Global Action Plan 2014-2019 promoted the implementation of integrated national eye health policies, plans and programmes; however, more work is needed for effective integration in all countries. ${ }^{15}$ Eye care is not typically included in health sector strategic plans, especially in LMICs. ${ }^{15}$ The lack of integration of DR services in health sector strategic plans means it has been excluded from the national planning and budgeting of services in the past. ${ }^{15}$

WHO has developed a tool which represents information for each country in terms of their non-communicable disease (NCD) progress monitoring indicators. ${ }^{16}$ The reports highlight which countries have implemented national integrated NCD policies, strategies or action plans. ${ }^{16}$ More specifically, the International Diabetes Federation (IDF) has developed a 'scorecard' which is a powerful tool for tracking DM country progress. ${ }^{17}$ The scorecard highlights how far countries have come in terms of implementing national DM plans and adopting a global monitoring framework for NCDs. ${ }^{17}$ There is no information on DR services available in the scorecards; therefore, making recommendations of inclusion of DR in DM/NCD planning would be useful to stimulate effective action to control sight loss due to DR in LMICs. A policy brief by Richardson $e t a l^{18}$ reported that many countries in Europe have incorporated specific DM national plans or included DM in their broader NCD strategies; however, limited information is available for LMICs. The DR Barometer Study provides a snapshot of global diabetes eye-related healthcare in a self-selected population of low-income countries, middle-income countries and high-income countries. ${ }^{19}$ The Barometer Study found that limited published research on global and country-specific awareness, prevention and effective management of DR is available, particularly in LMICs. ${ }^{19}$ This study also highlighted that a national strategy should be defined to ensure that people with DM are able to access eye examinations that are affordable to all. ${ }^{19}$

\section{STUDY RATIONALE}

The rationale for this scoping review is to provide a summary of which LMICs include DR in their national diabetes plans, and/or have begun DR screening, so that key stakeholders, including governments, multinational and non-governmental organisations can learn from them. The review aims to identify countries that have not begun such works, in order to focus resources on these countries and act as a benchmark for the future development of DESPs, to prevent DR-related blindness and visual impairment. Another reason for conducting this scoping review is to provide a map of the range of available evidence prior to conducting a large survey to identify more in-depth information on existing DESPs in LMICs. To our knowledge, no previous research has been conducted, which has prompted this scoping review.

\section{STUDY OBJECTIVES}

This scoping review has the following specific objectives:

- To identify LMIC settings that have not included DR services in their national DM strategic plans, action plans or policies, or as part of their NCD policies or prevention of blindness plans.

- To assess gaps in national level DR services planning in LMICs.

\section{METHODS AND ANALYSIS \\ Protocol design}

A scoping review requires a broad question to identify gaps in the existing literature and are intended to inform further investigation. This scoping review will include all study designs and grey literature due to the paucity of existing literature on DR screening in LMICs. Methods for this scoping review were developed based on the Arksey and O'Malley methodological framework, which were enhanced and expanded on by Levac et al. ${ }^{20}{ }^{21}$ Recommendations suggested by Joanna Briggs Institute will also be applied to increase clarity. ${ }^{22}$ The scoping review will be closely aligned to these frameworks and will consist of six stages as outlined below. This scoping review will be conducted in accordance with Preferred Reporting Items for Systematic Review and Meta-Analysis extension for Scoping Review (PRISMA-ScR) guidelines. ${ }^{23}$

\section{Stage 1: identifying the research question}

The research question was developed based on familiarity with a selection of existing literature and the inputs of established researchers in the field. The primary research question has been defined as, "which LMICs have included DR in their national DM, NCDs or prevention of blindness plans, and have or are developing DESPs'. This gives a clearly articulated scope of enquiry while remaining sufficiently broad.

\section{Stage 2: identifying relevant studies—-search strategy}

This scoping review will be as comprehensive as possible in identifying data (published and unpublished) from 
October 1989 (St. Vincent Declaration) to February 2020. To identify relevant databases, discussions with an academic librarian and well-established researchers in the field were had. The primary sources to use are Medline (Ovid), Embase (Ovid) and CENTRAL (Cochrane Library). Websites of relevant organisations, IDF, WHO, International Agency for the Prevention of Blindness and country-wide ministry websites will be searched for relevant reports or information. (The proposed search strategy is shown in online supplemental appendix, table 1)

Based on advice from an academic librarian and reviewing examples of relevant studies to determine commonly employed terminology, titles, abstracts and index terms used to describe a range of articles will be analysed. The main aim is to capture the widest selection of evidence on the topic through the search strategy.

\section{Stage 3: study selection}

The third stage of the Arksey and O'Malley's framework is to identify studies that will be included in the scoping review. Studies will be selected according to the data extraction framework adapted by Joanna Briggs Institute for scoping reviews ${ }^{22}$ (see supplemental appendix, table 2). As outlined below, this search is focused solely on LMICs, to determine which countries have or are developing DESPs for their populations with DM. ${ }^{24}$ Searches to determine which LMICs have included DR in their national DM, NCD or prevention of blindness plans or policies will be made, and grey literature will be particularly important for this study. Two reviewers (KC, PP) will independently screen titles and abstracts, cross-referencing the results. Titles and abstracts that do not meet the eligibility criteria will be excluded, and full-text articles will be retrieved for those that do meet the criteria. Disagreements about study eligibility will be discussed between the two reviewers until a consensus is reached. A PRISMA flow diagram (figure 1) will be developed to support the process of study selection.

\section{Inclusion criteria}

- Studies that had been conducted in LMICs to generate evidence to inform the development of national or subnational level DR screening and treatment programmes.

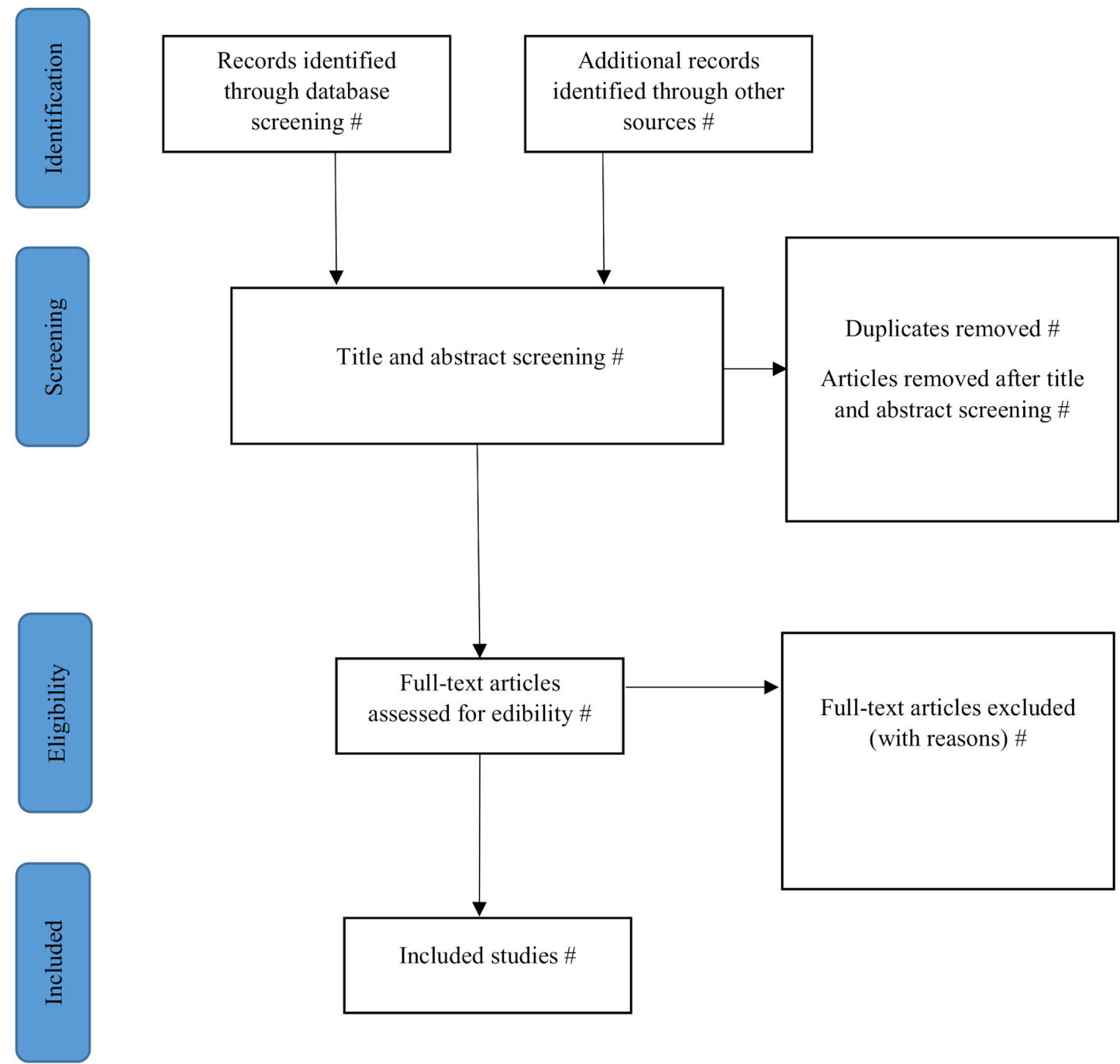

Figure 1 Preferred Reporting Items for Systematic Review and Meta-Analysis flow diagram: proposed search strategy and screening process. 
- Published articles/action plans/policy documents in LMICs on DM, NCD or DR that describe strategies of DR screening and treatment services at the national or sub-national level.

- Published articles/reports/policy documents in LMICs on eye care that describe strategies for the prevention of blindness and visual impairment due to DR.

\section{Exclusion criteria}

- Studies conducted before the St Vincent declaration (before 1989).

- Studies conducted in HICs.

- Studies not published in English.

\section{Stage 4: charting the data}

The data will be sorted according to the key themes and issues using thematic framework analysis. Co-reviewers (KC, PP) will be responsible for independently charting the data from each study included in the review. To ensure there is good inter-rater agreement between the reviewers, a subset of the included articles (10\%) will be assessed. Any discrepancies will be discussed by both reviewers until consensus has been reached. If the reviewers cannot agree on some studies, a third reviewer (arbitrator) will be necessary.

\section{Stage 5: collating, summarising and reporting the results}

A quantitative analysis will be carried out to map the data in tabular form, highlighting country progress in terms of national DESP implementation. The most updated evidence for a country will be used, and a colour-coded system, similar to the IDF scorecards will highlight country progress (red=no implementation, yellow=partial implementation and green=full implementation). Frequencies will be used to highlight the 'key findings' in the quantitative analysis and will be defined by country income level. The key findings will include (a) the number of countries who have a DM or NCD policy, (b) whether DR has been included in a DM or NCD policy and (c) whether countries have a national level eye care policy and whether DR is part of national eye care policy. A policy cycle approach to address gaps in national level DR service planning will be used, and a separate colour-coded system will highlight country progress in terms of their development in the policy cycle.$^{25} \mathrm{~A}$ thematic analysis will be used to identify emerging themes and subthemes from the included studies to guide a narrative summary of the literature. ${ }^{26}$ Thematic analysis will be conducted to identify the patterns of level of implementation of DR screening policies by country income setting based on the four main domains described in the policy cycle approach (ie, agenda setting, policy formation, policy implementation and evaluation). The key themes will highlight countries progress in terms of national level DR service planning and DESP implementation.

Stage 6: consultation

Consultation is an optional stage; however, to add methodological rigour to the study, this stage will be included.
The relevant stakeholder/s such as national government organisations, that is, Ministry of Health, will be contacted to offer additional sources of information, perspectives and meaning to the scoping review. These key informants will help to address gaps in the results to answer the main objectives of the study. These findings will help to inform the development of policies in DR and future research.

\section{Patient and public involvement}

Patients and the public were not involved in this protocol. This research will benefit people with diabetes in LMICs in order to provide equitable DR screening and treatment services to achieve universal coverage.

\section{Ethics and dissemination}

The results of this study will be disseminated through peer-reviewed journals and national and international conferences. By identifying gaps in the current body of literature, this study can guide future DR screening research and inform governments in LMICs. Since the methodology applied consists of reviewing and collecting data from publicly available resources, ethical approval is not required.

The proposed scoping review will identify countries who have not included DR in their national health plans, with the aim to inform government bodies and leading national health services. The findings from this scoping review will support improved access to diabetes-related eye care and promote global health equity. In many LMICs, lack of funding and implementation of relevant services can be problematic. Limited manpower and infrastructure to diagnose and monitor patients with DM and its complications pose great challenges. Establishing appropriately funded national level policies or plans that target reducing the impact of DM and DR is likely to be advantageous when coupled with adequate resource allocation, support and effective leadership. While addressing the DM-related and DR-related challenges is not a simple task, contributions from all key stakeholders (governments, healthcare providers, people with DM and societies) could potentially reduce the burden of the disease on the individual, their carers and on society. Based on the results of this scoping review, further research and advocacy work will be required to achieve the intended impact, so ministries of health implement strategies to improve access to diabetic eye care for people with DM. This scoping review will provide a platform for a comprehensive systematic review.

\section{Author affiliations}

${ }^{1}$ Centre of Public Health, Queen's University Belfast School of Medicine Dentistry and Biomedical Sciences, Belfast, UK

${ }^{2}$ Ministry of Health, Directorate of Policy Analysis and Development, Colombo, Sri Lanka

${ }^{3}$ Ophthalmology and Public Health, Sun Yat-Sen University Zhongshan Ophthalmic Center, Guangzhou, China

${ }^{4}$ Policy and Programmes, International Diabetes Federation, Brussels, Belgium ${ }^{5}$ Belfast Health and Social Care Trust, Belfast, UK 
Acknowledgements The authors would like to thank Hazel Neale, the librarian at Queens University Belfast for her support in developing the proposed search terms. The authors would also like to thank Dr Lynne Lohfeld for contributing to the final version of the protocol.

Contributors KC, PP, NC, TP, LD and BM aided in developing the research question and study methods, contributed meaningfully to the drafting and editing and approved the final protocol manuscript.

Funding NC is supported by the Ulverscroft Foundation (UK) and this publication is part of a thesis project.

Competing interests None declared.

Patient and public involvement Patients and/or the public were not involved in the design, or conduct, or reporting, or dissemination plans of this research.

Patient consent for publication Not required.

Provenance and peer review Not commissioned; externally peer reviewed.

Open access This is an open access article distributed in accordance with the Creative Commons Attribution Non Commercial (CC BY-NC 4.0) license, which permits others to distribute, remix, adapt, build upon this work non-commercially, and license their derivative works on different terms, provided the original work is properly cited, appropriate credit is given, any changes made indicated, and the use is non-commercial. See: http://creativecommons.org/licenses/by-nc/4.0/.

\section{ORCID iDs}

Katie Curran http://orcid.org/0000-0002-7071-109X

Prabhath Piyasena http://orcid.org/0000-0002-0236-0101

Tunde Peto http://orcid.org/0000-0001-6265-0381

\section{REFERENCES}

1 International Diabetes Federation. Global fact sheet, 2019. Available: https://www.idf.org/aboutdiabetes/what-is-diabetes/facts-figures. html [Accessed 10 Nov 2019].

2 International Diabetes Federation. IDF diabetes atlas. 9th edn, 2019. https://www.diabetesatlas.org/en/resources/

3 Cohen SR, Gardner TW. Diabetic retinopathy and diabetic macular edema. Dev Ophthalmol 2016;55:137-46.

4 Lee R, Wong TY, Sabanayagam C. Epidemiology of diabetic retinopathy, diabetic macular edema and related vision loss. Eye Vis 2015;2:1-25.

5 World Health Organization. Data and statistics the challenge of diabetes. Available: http://www.euro.who.int/en/health-topics/ noncommunicable-diseases/diabetes/data-and-statistics [Accessed 10 Mar 2020].

6 Yau JWY, Rogers SL, Kawasaki R, et al. Global prevalence and major risk factors of diabetic retinopathy. Diabetes Care 2012;35:556-64.

7 Cho NH, Shaw JE, Karuranga S, et al. IDF diabetes atlas: global estimates of diabetes prevalence for 2017 and projections for 2045. Diabetes Res Clin Pract 2018;138:271-81.

8 Diabetes Control and Complications Trial Research Group, Nathan DM, Genuth S, et al. The effect of intensive treatment of diabetes on the development and progression of long-term complications in insulin-dependent diabetes mellitus. N Engl J Med 1993;329:977-86.

9 Kohner EM, Aldington SJ, Stratton IM, et al. United Kingdom prospective diabetes study, 30: diabetic retinopathy at diagnosis of non-insulin-dependent diabetes mellitus and associated risk factors. Arch Ophthalmol 1998;116:297-303.

10 Stefánsson E, Bek T, Porta M, et al. Screening and prevention of diabetic blindness. Acta Ophthalmol Scand 2000;78:374-85.

11 Scanlon $\mathrm{PH}$. The English national screening programme for diabetic retinopathy 2003-2016. Acta Diabetol 2017;54:515-25.

12 Scanlon $\mathrm{PH}$. Screening intervals for diabetic retinopathy and implications for care. Curr Diab Rep 2017;17:96.

13 Piyasena MMPN, Murthy GVS, Yip JLY, et al. Systematic review on barriers and enablers for access to diabetic retinopathy screening services in different income settings. PLoS One 2019;14:e0198979.

14 World Health Organisation. World report on vision, 2019. Available: https://www.who.int/publications-detail/world-report-on-vision [Accessed 16 Feb 2020].

15 The International Agency for the Prevention of Blindness. Global action plan 2014-2019, 2020. Available: iapb.org/advocacy/globalaction-plan-2014-2019/ [Accessed 2 Mar 2020].

16 World Health Organisation. Noncommunicable disease progress monitor, 2020. Available: who.int/publications-detail/ncd-progressmonitor-2020 [Accessed 10 Mar 2020].

17 International Diabetes Federation. Global diabetes Scorecard, 2020. Available: https://idf.org/our-activities/advocacy-awareness/ resources-and-tools/53-global-diabetes-scorecard.html [Accessed 13 Feb 2020].

18 Richardson E, Zaletel JM, Nolte E. National diabetes plans in Europe: what lessons are there for the prevention and control of chronic diseases in Europe, 2016. Available: http://www.euro.who.int/_data/ assets/pdf_file/0009/307494/National-diabetes-plans-Europe.pdf [Accessed 17 Feb 2020].

19 Cavan D, Makaroff L, da Rocha Fernandes J, et al. The diabetic retinopathy barometer study: global perspectives on access to and experiences of diabetic retinopathy screening and treatment. Diabetes Res Clin Pract 2017;129:16-24.

20 Arksey H, O'Malley L. Scoping studies: towards a methodological framework. Int J Soc Res Methodol 2005;8:19-32.

21 Levac D, Colquhoun H, O'Brien KK. Scoping studies: advancing the methodology. Implement Sci 2010;5:1-9.

22 The Joanna Briggs Institute. The Joanna Briggs Institute Reviewers' Manual 2015: Methodology for JBI scoping reviews, 2015. Available: https://nursing.Isuhsc.edu/JBI/docs/ReviewersManuals/Scoping-.pdf [Accessed 28 Jan 2020].

23 Tricco AC, Lillie E, Zarin W, et al. PRISMA extension for scoping reviews (PRISMA-ScR): checklist and explanation. Ann Intern Med 2018;169:467-73.

24 The World Bank. World bank country and lending groups, 2020. Available: ttps://datahelpdesk.worldbank.org/knowledgebase/ articles/906519-world-bank-country-and-lending-groups [Accessed 18 May 2020].

25 Weible CM, Heikkila T, deLeon P, et al. Understanding and influencing the policy process. Policy Sci 2012;45:1-21.

26 Ritchie K, Spencer L. Qualitative data analysis for applied policy research. In: Bryan A, Burgess RG, eds. Analysing qualitative data. London: Routledge, 1994: 173-94. 